\title{
AIR QUALITY AND POLLUTION MAPPING SYSTEM, USING REMOTE MEASUREMENTS AND GPS TECHNOLOGY
}

\author{
C. SCHREINER \\ M. BRANZILA \\ A. TRANDABAT \\ R.C. CIOBANU*
}

\author{
Technical University "Gh. Asachi” of lasi \\ Faculty of Electrical Engineering \\ Electrical Measurements Dept. \\ Bdul. D. Mangeron No. 53-55 \\ 700050, IASI, Romania
}

Received: $13 / 02 / 06$

Accepted: 30/05/06 *to whom all correspondence should be addressed: e-mail: rciobanu@ee.tuiasi.ro

\begin{abstract}
According to the actual European context, including the development of risk free residential and industrial areas, the main demand lies in instant, accurate and elaborated information regarding environment quality and pollution risks.

This paper presents a conceptual architecture for a versatile, flexible, cost efficient, highspeed instrument for monitoring the air quality.

In order to cover a large monitoring area, the system is placed in the luggage compartment of a glider. The glider was peculiarly selected, because, in free flight, it covers a wide area hundreds of kilometers per hour - with a reasonable speed in an economical way, may allow repetitive flight cycles at different altitudes, and, by comparing to a motorized aircraft, it caries the mobile part of the system without affecting the measurement precision by fast acceleration, vibrations, turbulences or polluting exhaust gases.
\end{abstract}

The device, composed by two main physic parts: the mobile and the field components, is remotely controlled, and no kind of intervention from pilot part is requested during the flight, meaning that the measurement operation may be completely accessed from the ground anytime during flight schedules and doesn't depend on pilot knowledge or action.

The mobile component is composed by an acquisition block, based on specialized gas sensors inter-connected within a prototype acquisition board, a positioning system which is represented by a GPS map 196, a laptop and a transmission module.

The software platform - in terms of virtual instruments - was developed under Labview programming environment.

The communication between GPS and laptop is realized by a RS232 interface, using the NMEA protocol. The information is dynamically processed, allowing both the survey and alert regimes, in order to allow the immediate pertinent decisions, directly, or by use of an expert/esurvey system.

The data related to air quality map for the monitored site should be further submitted to a statistical - pollution (contamination) process - control, allowing the evaluation of: peak, average, trend and prediction of pollution, related either to temporal (season, day/night, peak hours etc.) or geographical parameters (altitude, vicinity etc.), or to atmospheric conditions (humidity, wind etc.), or even to societal demands (fuel type, production planning, urban development, or other relevant contextual circumstances).

The immediate potential application lies in mapping the air pollution factors dispersion and cause identification, in order to answer to the needs of residential and industrial areas sustainable expansion, a very important problem met mainly by candidate countries to EC.

KEYWORDS: remote measurements, GPS, virtual instrumentation, air quality surveillance, mobile mapping system attached to a glider. 


\section{INTRODUCTION}

The EU-funded conference on "Environment, Health, Safety: a challenge for measurements", held in Paris in June 2001, recognized the need to improve the performance of environmental measurement systems and their harmonization at EU level, to foster the dialogue between the providers of measurement methods and the users of measurement results, and to prepare the base - by establishing special communication tools - for the integration of research expertise and resources of environmental monitoring across Europe. The concept presented herein aims to respond to this actual challenge by combining the latest software trends with the newest hardware concepts in environmental monitoring, towards providing reliable measurement results and representative environmental indicators, evaluating trends and quantifying the achieved results in order to manage the potential environmental risk in compliance with European legislation and local particularities.

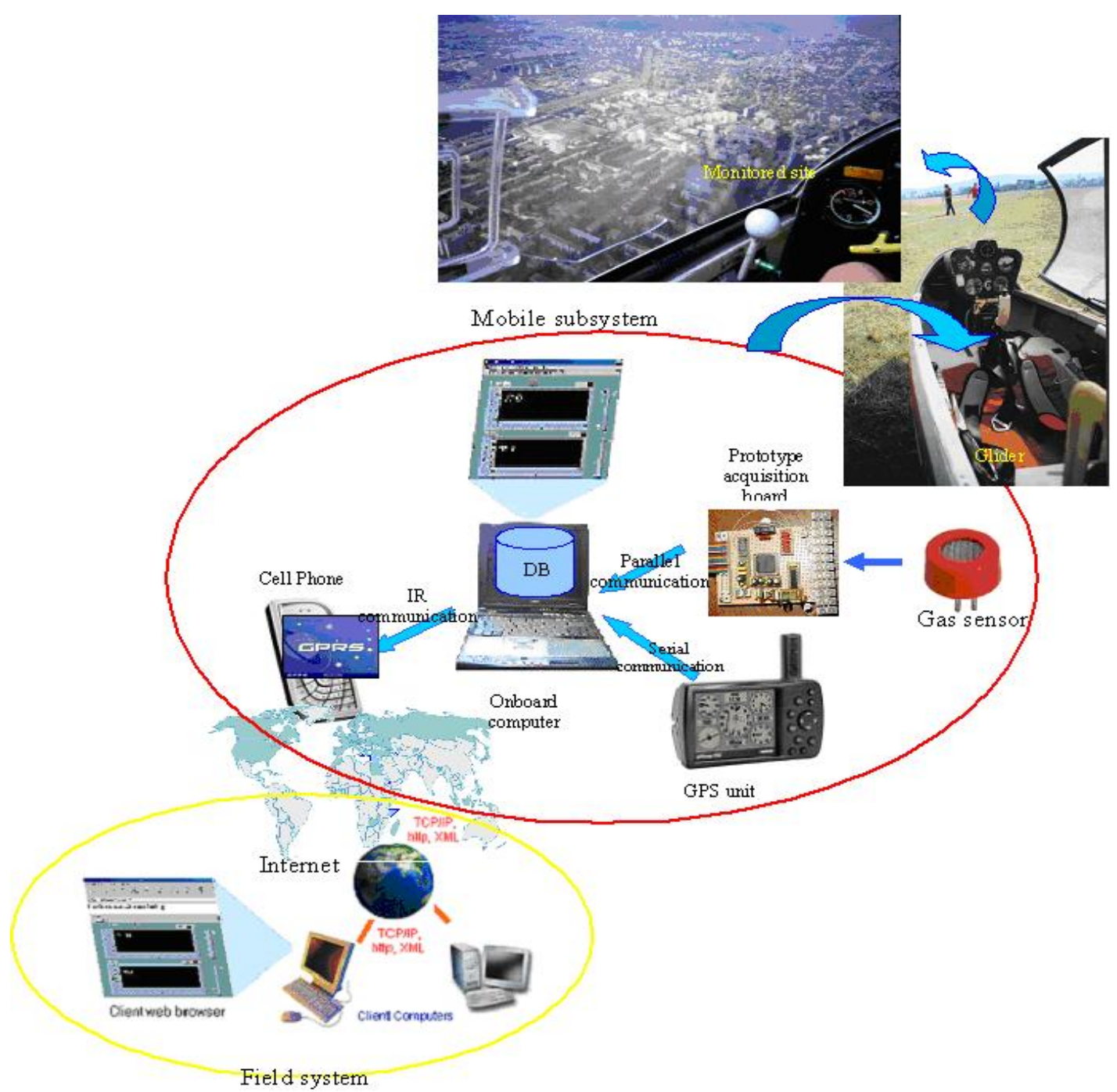

Figure 1. The concept system for air quality monitoring, using a prototype data acquisition board, GPS and GPRS technology

The decisions related to the environment safety are often taken in the belief that they are scientifically well founded, i.e. by placing an excessive faith in the reliability of the expert information on which they are based. But, during the last century, such a pursuit was denied by an alarming number of environmental injuries, causing a continuously growing societal concern. Today - more than ever - the public demands credible and understandable information about the quality of the environment in which they live or work, the trend of environmental indicators, the priority problems related to environment pollution and long term associated risks. Accordingly, the common uncertainty and/or ignorance in decision-making 
must be balanced by innovative and multidisciplinary methods in order to carry out an efficient exchange of information across the different sectors and aspects involved in environmental monitoring.

One of our main concerns is represented by the atmospheric environment, very sensitive to a synergy of factors, as consequence of three phenomena: global warming, ozone-layer depletion and, above all, local air pollution. Among all, global warming is the most critical in terms of environmental conservation, a clear result of greenhouse-gas emissions excess, mainly carbon dioxide $\left(\mathrm{CO}_{2}\right)$. Nevertheless, the first step towards environment quality conservation lies in monitoring efficiently the factors with potential risk at local dimension. The actual practice clearly demonstrated that the classical, local, static measurements are affected by uncertainties, even errors. These uncertainties are propagated through the models of complex systems and finally presented in some form to the decision-maker, with tragic consequences. Therefore, the need to revise the existing models by checking their compatibility with the precautionary principle of vertical and dynamic monitoring represents a must for the novel reliable measurement systems, [1, 2].

Such an example is offered by the remote measurement system described below.

\section{SYSTEM ARCHITECTURE}

The main objective of our research consisted in realizing the complex device for mapping the atmosphere quality parameters, composed by two main physic parts: the mobile and the field components (Figure 1). In order to cover a large monitoring area, the mobile system is placed in the luggage compartment of a glider. The glider was peculiarly selected, because, in free flight, it covers a wide area - hundreds of kilometers per hour - with a reasonable speed in an economical way, may allow repetitive flight cycles at different altitudes, and, by comparing to a motorized aircraft, it caries the mobile part of the system without affecting the measurement precision or traceability by fast acceleration, vibrations, turbulences or polluting exhaust gases.

The device is remotely controlled, and no kind of intervention from pilot part is requested. The mobile component is composed by: an acquisition block, based on specialized gas sensors inter-connected within a prototype acquisition board, a positioning system which is represented by a GPS map 196, a laptop and a transmission module. The software platform in terms of virtual instruments - was developed under Labview programming environment. The communication between GPS and laptop is realized by a RS232 interface, using the NMEA protocol.

Normally, the recorded data sent by GPS to the database, according to an Internet protocol, are the values corresponding to the longitude, latitude and altitude, and separately, for a further complex data analyze, the description of glider speed evolution. At the same time, the database receives also the values for the monitored gases concentration through a parallel port from the acquisition prototype board where the sensors are connected. The information is dynamically processed, allowing both the survey and alert regimes. The survey (passive) transmission allows the system data to be remotely stored via an Internet protocol in the database, from where they may be processed off-line, becoming available and useful later for the decisional factors, local authorities, public and monitoring/accreditation bodies. The active (alert) mode processes the real time information in terms of neuro-fuzzy decisions, according to contextual circumstances. If any of monitored parameters is discovered to be beyond the accepted values, the communication module is activated and a warning message (including cause, position and recorded value) is sent by use of a normal GPRS mobile phone to the responsible authority, for an immediate decision. This real-time information from the monitored sites can determine an immediate pertinent reaction, taken either directly by the person in charge, or by use of an expert system, [3-6].

The mobile system autonomy depends on the laptop accumulator power and type, being normally limited to three hours of continuum monitoring. But, taking into account that the normal glider flight doesn't exceed this period, and mainly, that this kind of measurements are either emergency, comparative, or for mapping purposes, the system fulfills successfully its technical destination. Nevertheless, if more or longer time measurements are needed, only batteries must be changed, according to the flight schedule, that may be even adapted from amateur flight to dedicated survey. 
For common communication procedure between the measurement point assisted by a laptop and the server, DataSoket communication and TCP/IP tools were preferred. The expert user from the ground has the possibility to visualize the real data and/or to analyze the environmental quality factors via a history diagram stored in the server database.

The bloc diagram of the server virtual instrument is presented in figure 2. All communication software is designed under LABVIEW graphical programming language as well, including three protocol types. Communication type PC-instruments is developed using GPIB protocol, PC-server using TCP/IP and Internet communication using data socket technology, (see also figure 1), [6].

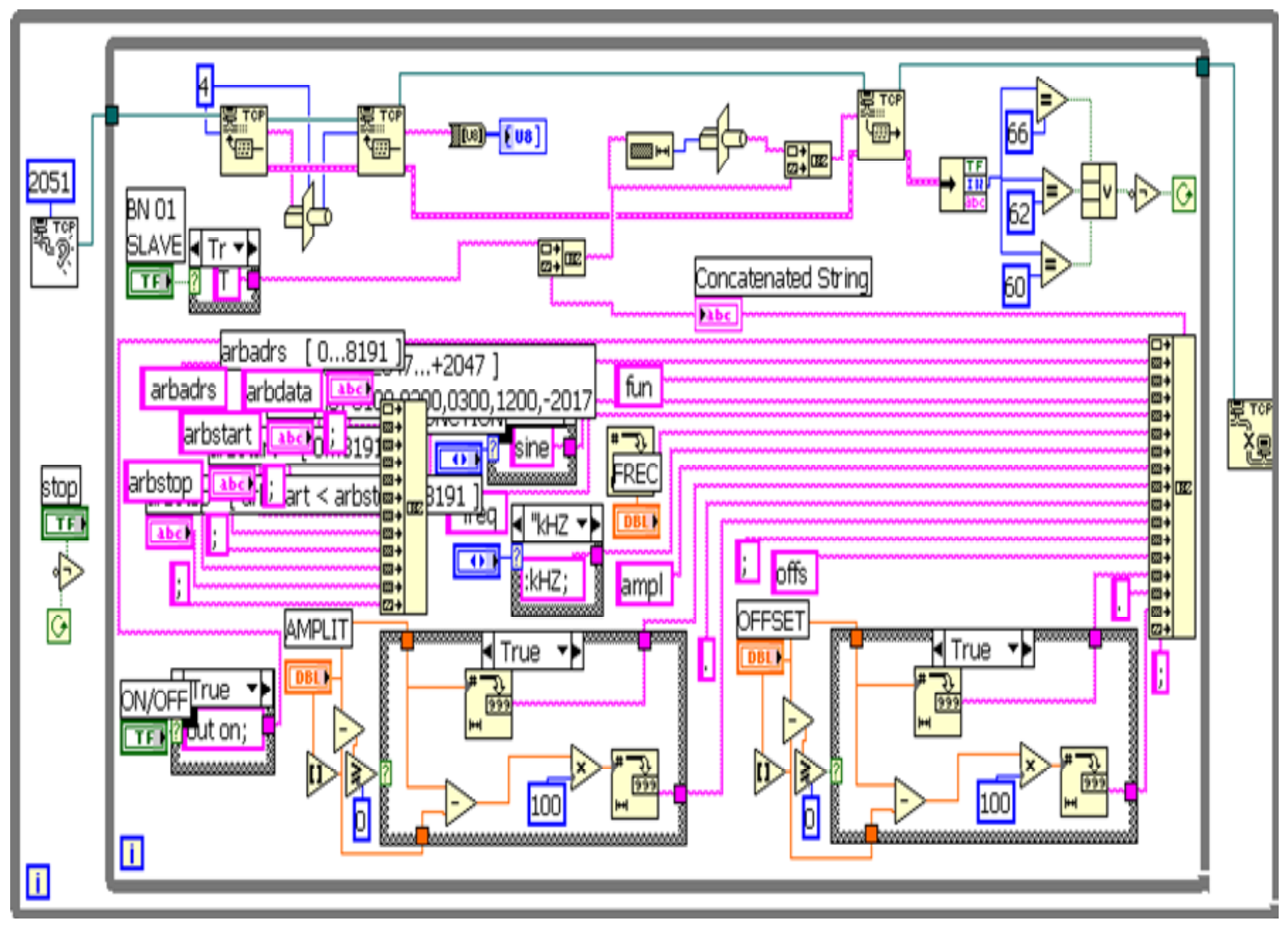

Figure 2. Server bloc diagram

\subsection{Data acquisition board}

The LM12H458CIV chip was preferred to realise a Data Acquisition Board for interfacing with the PC by the parallel port. The results are a portable Data Acquisition Systems - DAS and a flexible and complex system that was used to allow monitoring of the environmental parameters. The drivers and the virtual instruments were created under LabVIEW software.

The board is related to highly integrated DAS. Operating on just $5 \mathrm{~V}$, it combines a fully differential self-calibrating (correcting linearity and zero errors) 13-bit (12-bit + sign) analogueto-digital converter (ADC) and sample-and-hold $(\mathrm{S} / \mathrm{H})$ with extensive analogue functions and digital functionality. Up to 32 consecutive conversions using two's complement format it is stored in an internal 32-word (16-bit wide) FIFO data buffer (figures 3 and 4). An internal 8word RAM can store the conversion sequence for up to eight acquisitions through the LM12H458CIV's eight-input multiplexer. The LM12H458CIV operates with 8-bit + sign resolution and in a supervisory "watchdog" mode that compares an input signal against two programmable limits, $[7,8]$. 


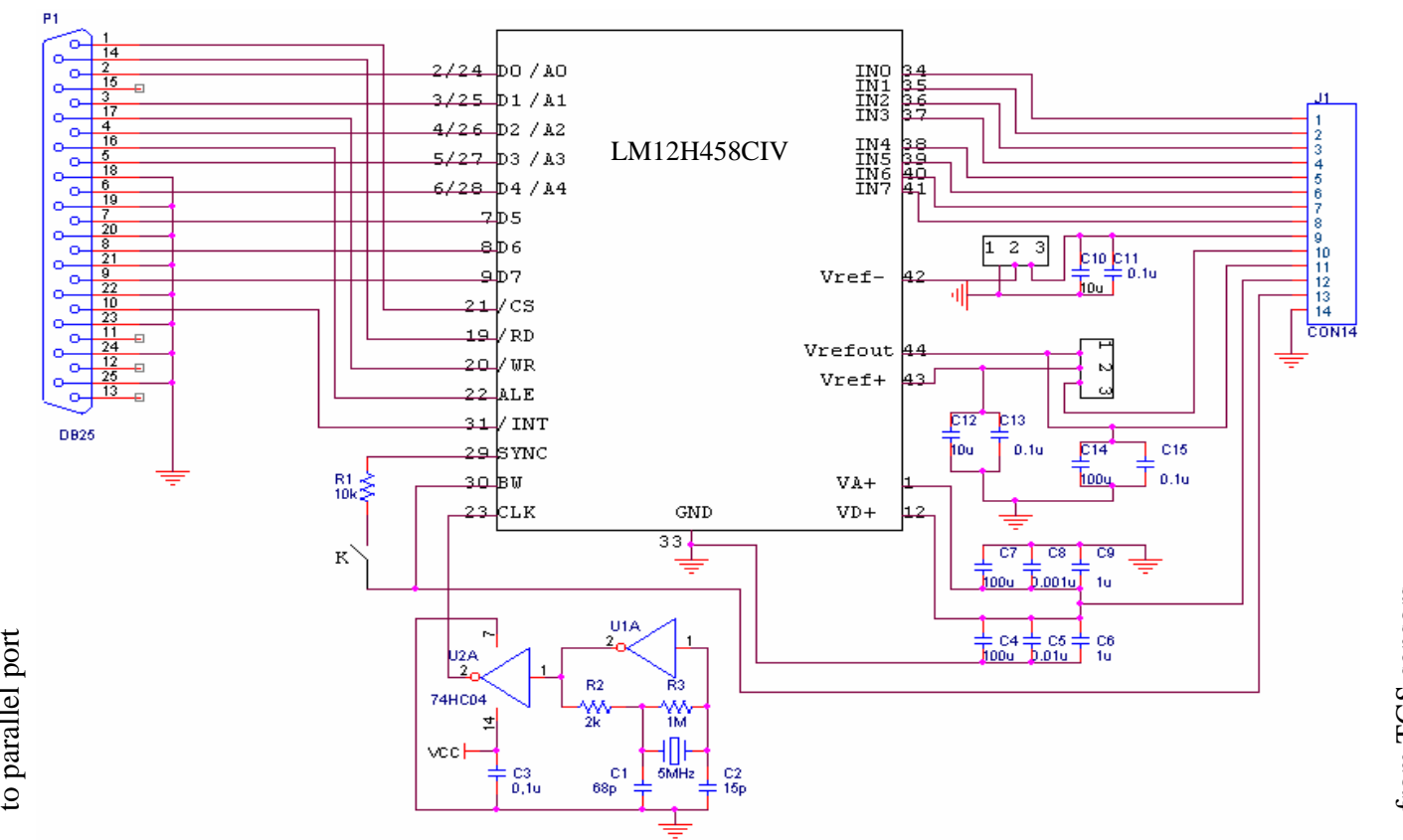

Figure 3. Architecture of data acquisition board, through parallel port of PC

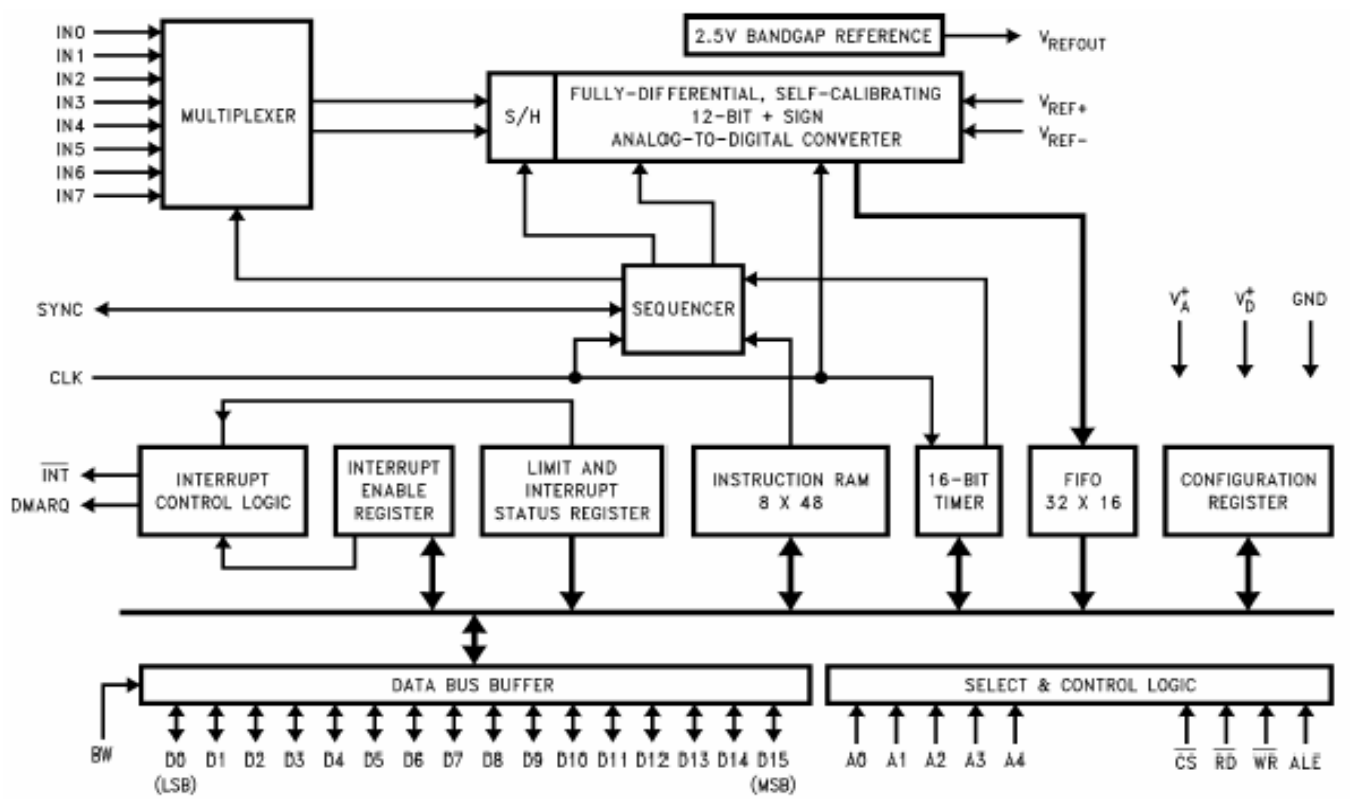

Figure 4. Functional diagram for the chip LM12H458CIV / Data Acquisition System.

Programmable acquisition times and conversion rates are possible through the use of internal clock-driven timers. The reference voltage input can be externally generated for absolute or ratiometric operation or can be derived using the internal $2.5 \mathrm{~V}$ bandgap reference. All registers, RAM, and FIFO are directly addressable through the high-speed microprocessor interface to either an 8-bit or 16-bit databus. The LM12H458CIV include a direct memory access (DMA) interface for high-speed conversion data transfer.

Based on the bloc diagram of the server virtual instrument, presented in figure 2 , the frame of virtual instruments directory is presented in Figure 5. LabVNC (based on the VNC - Virtual Network Computing protocol) allows the instantly enabling of web control upon virtual instruments VI. Accordingly, LabVNC creates a Java applet, an exact representation of any VI selection, giving full control of the selected VI. LabVNC consists of a VI control panel, 
dedicated DLLs and a binary executable, that is the LabVNC server. Currently, the system runs on 32-bit Windows and requires LabVIEW 6.0 or greater.

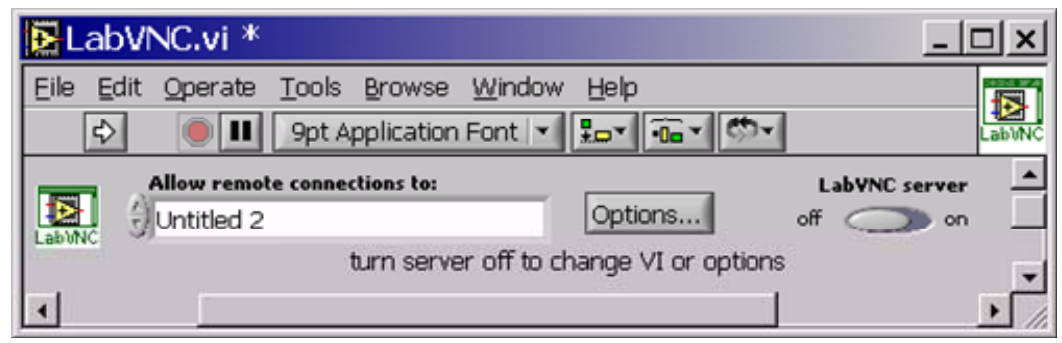

Figure 5. LanVNV front panel

\subsection{Detection circuit}

In our application, dedicated sensors for temperature and gas evaluation (sensing element metal oxide semiconductor, mainly composed of $\mathrm{SnO}_{2}$ ) for air quality analysis were used. In principle, mainly the gas sensors should be of highest quality, because they should offer immediate pertinent information towards defining representative environmental indicators, allowing evaluation of trends and quantification of achieved results in connection with temporal (season, day/night, peak hours etc.) or geographical parameters (altitude, vicinity etc.), or to atmospheric conditions (humidity, wind etc.), or even to societal demands (residential or industrial areas), [9-10]. The sensing element is heated at a suitable operating temperature by a built-in heater, allowing a sensitive change in its electrical resistance. In pure air, the sensor resistance is high, but, when exposed to a variety of gases, the sensor resistance decreases selectively in accordance with the gas type and concentration (figure 6). Based on this information, the expert system from ground server processes the data according to a statistical - pollution (contamination) process - control methodology, decrypting the gases type and concentration and mapping the potential risk for environment safety. Figure 7 presents the basic detection circuit. The change in the sensor resistance is obtained as the change of the output voltage across the load resistor $\left(R_{L}\right)$ in series with the sensor resistance. The constant $5 \mathrm{~V}$ output of the data acquisition board is available for the heater of the sensor $\left(\mathrm{V}_{\mathrm{H}}\right)$ and for the detecting circuit $\left(\mathrm{V}_{\mathrm{C}}\right)$. As already indicated, the LM12H458CIV chip was preferred to make a Data Acquisition Board for interfacing with the laptop by parallel port and realise a flexible and complex system to allow monitoring of environmental parameters via some detection circuits with gas sensors distributed around the glider.

\subsection{Application and further development}

A pilot research cooperation was established in 2005 with the kind support from the Local Council, Town Hall and the local Environmental Agency in lasi. The 10 months research is still under development, and the target lies in mapping all the residential and industrial areas of the county, improving the remote instruments and developing the expert system.

An example of end product is presented in figure 8 , for a residential area in the vicinity of an industrial one and close to the city electro-thermal station (on coal fuel). The data will be further processed according to a statistical - pollution (contamination) process - control, allowing the evaluation of: sort and impact (gases type and concentration), peak, average, trend and prediction of pollution, related either to temporal (season, day/night, peak hours etc.) or geographical parameters (altitude, vicinity etc.), or to atmospheric conditions (humidity, wind etc.), or even to societal demands (fuel type, production planning, urban development, or other relevant contextual circumstances). Such an expert system is in work at this time, and will process off-line the data towards an active database, even corroborated with other information obtained previously or independently from the (autonomous) meteorological stations or balloons, or from the ground sensors. By this way, the system primarily receives real-time information from the monitored sites, evaluates/predicts the potential risk for environment safety and can generate automatic reports to the most appropriate control centers, from where eventually a potential pertinent intervention can be done. 


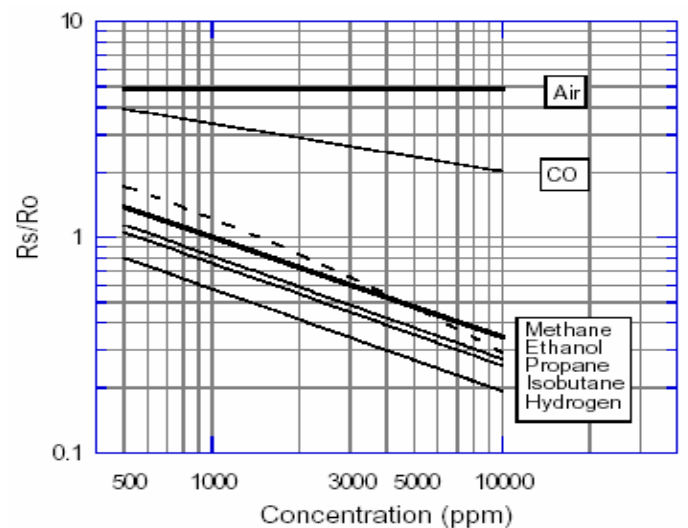

Figure 6. Sensitivity characteristics

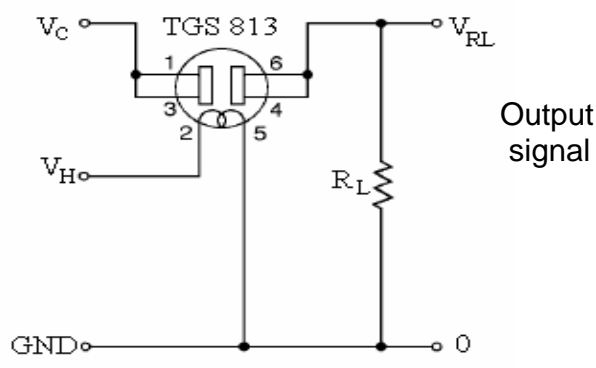

Figure 7. Detection circuit

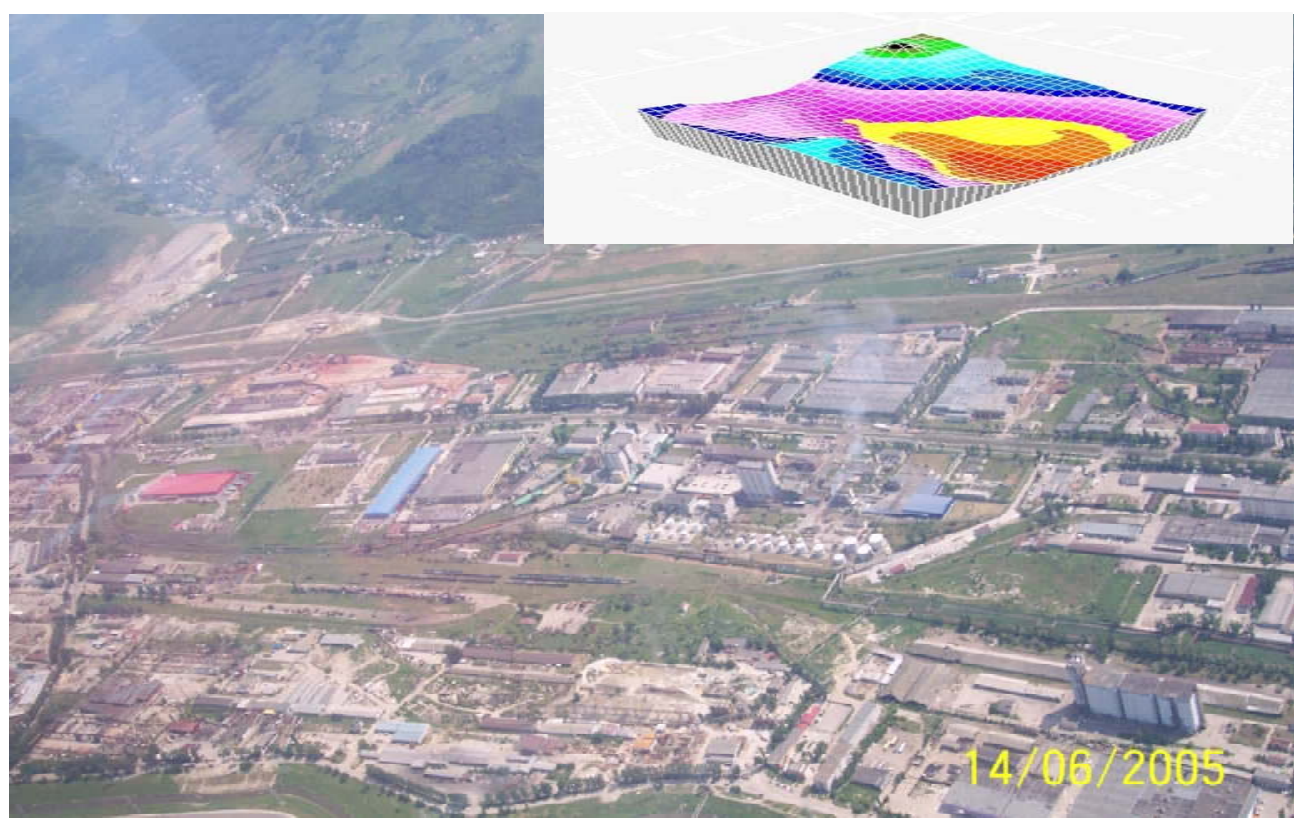

Figure 8. Example of 3D air quality map; with dark red - the major risk area

But, nevertheless, the proposed concept may be very useful not only for the decisional factors, local authorities, accreditation bodies etc., but also for the educational process and public aware. The proposed concept was tested already as an educational tool for the students dealing with 'Environment Quality and Maintenance Management' discipline, representing in fact a task of a Virtual Laboratory for higher education purpose in environment safety field, as described in $[1,11]$.

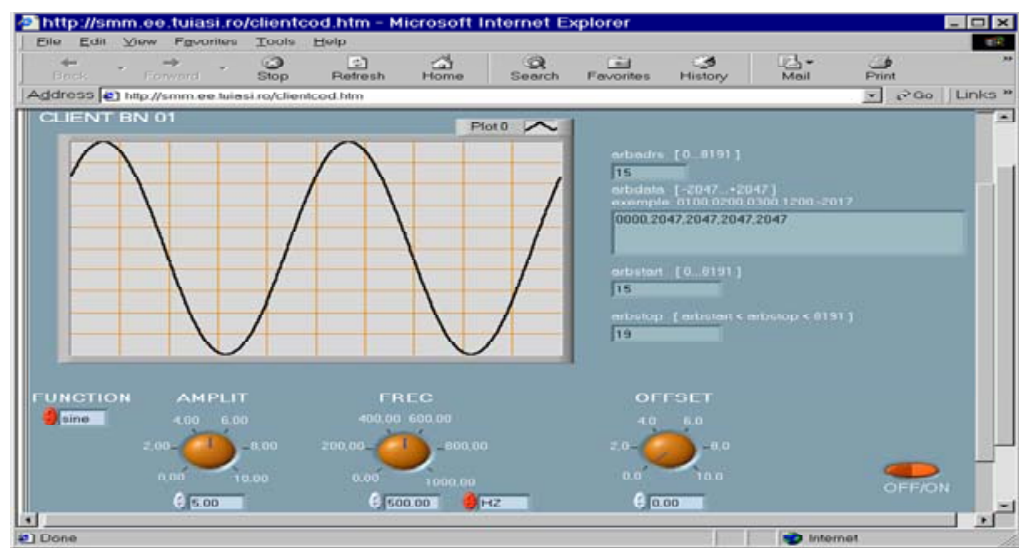

Figure 9. Remote system frond panel under Explorer 


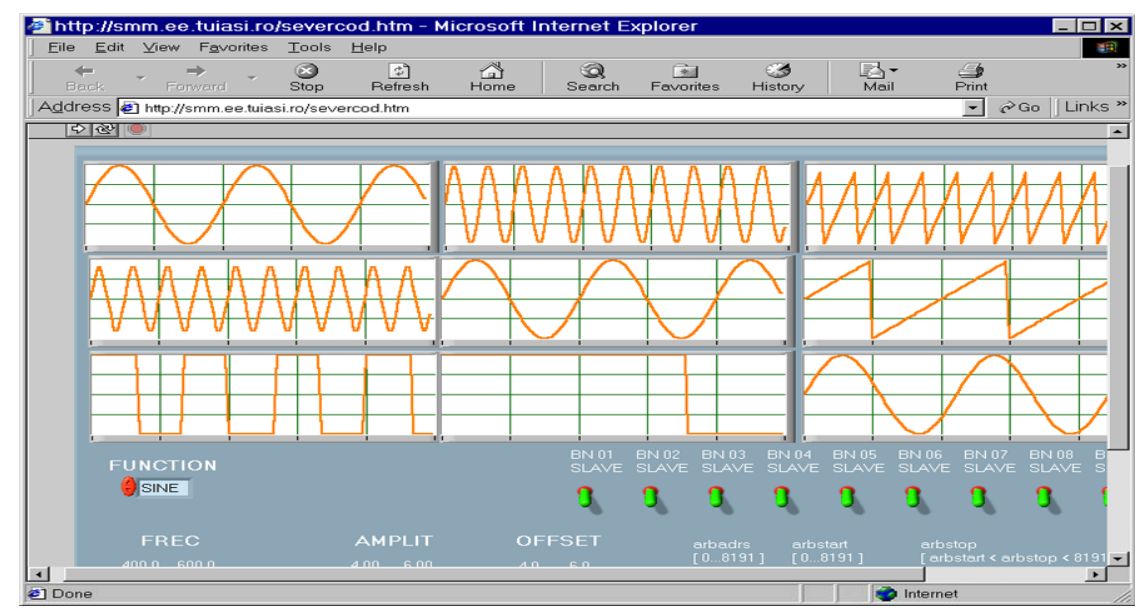

Figure 10. Server panel under Explorer

Accordingly, an example of front panels of an application on both server and remote system (sensors signal acquisition and conditioning) is presented in figures 9 and 10, for Internet Explorer browser (http://www/smm.ee.tuiasi.ro/servercode.htm).

\section{CONCLUSIONS}

The paper presents the architecture of a versatile, flexible, cost efficient, high-speed instrument for either monitoring the air quality and/or mapping the air pollution. The concept is based on a remotely controlled acquisition part - placed in a glider - with distributed and virtually programmed gas sensors, and a local dedicated expert system.

The system may be particularised as virtual laboratory for on-line environmental monitoring classes too, helping the formation of well trained specialists in the domain.

The immediate potential application lies in mapping the air pollution in terms of: factors, dispersion, trend, evolution and causes identification, in order to answer to the needs of immediate action and/or residential and industrial areas sustainable expansion, very important problems met mainly by candidate countries to EC.

\section{ACKNOWLEDGEMENT}

The authors thankfully acknowledge the kind technical support (regarding glider modification) of the Romanian Air Club - lasi branch. The measurements were performed personally by the third co-author (licensed pilot with R.A.C. - lasi branch).

\section{REFERENCES}

1. Benetazzo L., Bertocco M., Ferraris F., Ferrero A. and Piuri V. (2000) A Web-based distributed virtual educational laboratory, IEEE Trans. on Instrumentation and Measurement, 49(2), 349-356.

2. Bertocco M., Cappellazzo S., Carullo A., Parvis M. and Vallan A., (2001) Virtual environment for fast development of distributed measurement applications, IEEE International Workshop on Virtual / Intelligent Measurement Systems - VIMS 2001, May 2001, Budapest, Hungary, 57-60.

3. Winiecki W. and Karkowski M., (2001) A new Java-based software environment for distributed measurement systems, Proc. of IEEE IMTC 2001, May 2001, Budapest, Hungary, 397-402.

4. Ando B., Baglio S., Graziani S. and Pitrone N., (2000) Models for air quality management and assessment, IEEE Transactions on systems, man and cybernetics-part C: Applications and reviews, 30(3), XX-XX.

5. Arpaia P., Baccigalupi A., Cennamo F. and Daponte P., (2000) A measurement laboratory on geographic network for remote test experiments, IEEE Trans. on Instrumentation and Measurement, 49(5), 992-997. 
6. Donciu C., Cretu M. and Fosalau C., (2001) Communication in virtual instrumentation, Int. Conf. on Management of technological changes, Oct. 25-26, 2001, lasi, Romania, 69-74.

7. National Semiconductor, 'Technical Information for LM12458', July 1999.

8. Figaro Engineering Inc., Technical Information for TGS 813/813C, Revised June 1996.

9. Girao P., Postolache O., Pereira M. and Ramos H. (2003) 'Distributed measurement systems and intelligent processing for water quality assessment', Sensors \& Transducers Magazine, 38(12), 82-93.

10. Trandabat A., Pislaru M., Schreiner C. and Ciobanu R., (2005) E-survey instruments based on remote measurements dedicated to peculiar areas with increased risk for environmental safety, $9^{\text {th }}$ Int. Conf. on Environmental Science and Technology, Sept. 1-3, 2005, Rhodes, Greece, Vol. B, 933-938.

11. Trandabat A., Branzila M. and Schreiner C., (2005) Distributed measurements system dedicated to environmental safely, $4^{\text {th }}$ Int. Conf. on the Management of Technological Changes, Chania, Greece, August 19-20, 2005, Book 2, 121-124. 